\title{
Long-Term Mechanical Ventilation: Management Strategies
}

\author{
Alexander C White MD
}

\author{
Introduction \\ Weaning or Discontinuing PMV \\ Identifying and Correcting Barriers to Weaning \\ Special Situations \\ Systematic Approach to Weaning Trials/Liberation Trials \\ Failure to Wean and the Decision to Proceed With Life-Long Support \\ Tracheostomy Tube Management During PMV \\ How Does One Select a Ventilator for the LTAC, Compared to the ICU? \\ Summary
}

\begin{abstract}
This paper reviews management strategies for patients undergoing prolonged mechanical ventilation (PMV). Topics covered include how to identify and correct barriers to weaning, the systematic approach to weaning trials, when to cease weaning trials and proceed with life-long support, managing the tracheostomy tube during PMV, and, finally, how to select a suitable mechanical ventilator for PMV. Key words: long-term mechanical ventilation; prolonged mechanical ventilation; weaning; tracheostomy; mechanical ventilation. [Respir Care 2012;57(6):889-897. () 2012 Daedalus Enterprises]
\end{abstract}

\section{Introduction}

Prolonged mechanical ventilation (PMV) provides ventilatory support to patients with either isolated failure of the respiratory system or respiratory failure occurring as a component of chronic critical illness. ${ }^{1}$ In the absence of a consensus for the definition of PMV, a useful practical definition of PMV onset is the time of tracheostomy tube insertion for continued mechanical ventilation (MV). The

Dr White is affiliated with the Department of Pulmonary and Sleep Medicine, New England Sinai Hospital, Stoughton, Massachusetts.

Dr White presented a version of this paper at the 49th RESPIRATORY CARE Journal Conference, "The Chronically Critically Ill Patient," held September 9-10, 2011, in St Petersburg, Florida.

The author has disclosed no conflicts of interest.

Correspondence: Alexander C White MD, Department of Pulmonary and Sleep Medicine, New England Sinai Hospital, 150 York Street, Stoughton MA 02720. E-mail: awhite@nesinai.org.

DOI: $10.4187 /$ respcare. 01850 patient who requires PMV has, by definition, failed multiple attempts at weaning. The need to transfer the patient to a specialized weaning unit may be another way to identify patients who have transitioned from short-term MV to PMV. However, in some areas, patients who require PMV are sometimes managed in the ICU setting. For the purposes of this review we will define PMV as MV that is needed for at least 21 days.

\section{Weaning or Discontinuing PMV}

A recent consensus statement proposed categorizing patients into 3 groups, based on the duration of the weaning process, with patients who require PMV falling into group $3 .^{2}$ These patients have already failed multiple attempts to wean from MV and have required MV for at least 7 days.

A major goal of managing any patient who requires PMV is to establish if it is possible to liberate or wean the patient from the mechanical ventilator. Reducing the amount of time the patient has to spend on the ventilator substantially improves quality of life, by allowing phona- 
tion, enhancing functional mobility, and giving the patient a sense of independence. Reducing the number of days of MV may reduce the likelihood of ventilator-associated pneumonia.

The process of liberating the patient from PMV can be divided into 2 phases. During the first phase, physiological barriers to weaning are identified and corrected if possible. The second phase involves a systematic approach during the attempts to "wean" the patient from MV. When managing any patient who requires PMV, it is important that practitioners remain alert over time to improvements in lung compliance or respiratory muscle strength that may allow weaning to commence in a patient who has previously failed attempts to wean. ${ }^{2,3}$

\section{Identifying and Correcting Barriers to Weaning}

The major causes of failure to wean from PMV are linked to age, barriers to weaning (mainly cardiorespiratory disease), and comorbid conditions that further complicate barriers to weaning and nutrition. Our understanding of the aging process and its impact on failure to wean from PMV is limited. Age and the need for invasive MV have been associated with increased hospital mortality, after adjusting for severity of illness. ${ }^{4,5}$ Data from animals indicate that aging diminishes diaphragmatic muscle function, ${ }^{6}$ independent of the known negative effects of MV per se on diaphragm function. ${ }^{7}$ Age has also been associated with "anabolic resistance," resulting in increased muscle breakdown and reduced muscle synthesis following critical illness, ${ }^{8,9}$ which likely contributes to impaired diaphragm function. This may explain in part the relationship between age and failure to wean from PMV.

A number of conditions are a common occurrence in the population of patients who require PMV and are barriers to weaning. This subject has been previously reviewed in detail.2,3 Pulmonary disease makes up over $50 \%$ of patients who require PMV. Increased work of breathing, impaired respiratory drive, and inspiratory muscle weakness are the major factors. Whether or not the patient can be removed from MV will be determined by the ability of the patient's respiratory system to manage the imposed respiratory load. The load includes static and dynamic lung compliance, and to some extent the alveolar-arterial oxygen difference. Parenchymal lung disease reduces compliance from its normal value of $0.06-0.1 \mathrm{~L} / \mathrm{cm} \mathrm{H}_{2} \mathrm{O}$, and increases the work of breathing. Typical processes that reduce lung compliance include healthcare associated pneumonia, pulmonary edema, interstitial lung disease, and acute lung injury. These processes may also result in the need for PMV. Clinicians managing patients need to be alert to improvements in lung compliance and alveolar-arterial oxygen difference over time that may allow a patient on
PMV to begin the weaning process. Mechanical ventilators with graphic displays available in most weaning units ${ }^{10}$ allow clinicians to check static and dynamic lung compliance. Newer mechanical ventilators, along with other devices, can provide real time analysis of end-tidal $\mathrm{CO}_{2}$ and carbon dioxide production, which may help weaning from PMV.

Airways disease is a major cause of PMV. ${ }^{11}$ COPD is the most common disease process in this category and increases work of breathing through air-flow obstruction, which in turn may also cause dynamic hyperinflation and auto-PEEP. Other less common airway diseases that may result in PMV include cryptogenic organizing pneumonia, tracheomalacia, chronic asthma, and airway complications of lung and stem cell transplantation, such as bronchiolitis obliterans. Optimizing bronchodilator treatment, judicious use of airway stents, and dilatation of stenotic segments of airway at anastamotic sites may help improve dynamic compliance to the point that weaning may become possible in some of these patients. Unfortunately, some pulmonary disease processes are irreversible, such as end stage COPD and neuromuscular disease, and in these cases PMV becomes a life-long treatment option.

Congestive heart failure or ischemic heart disease is reported in up to $26 \%$ of patients admitted to long-term acute care (LTAC) hospitals for weaning from PMV. ${ }^{11} \mathrm{By}$ the time a patient has transitioned to PMV, cardiac function has usually been optimized. However, spontaneous breathing trials (SBTs) during the weaning process may uncover cardiac dysfunction by increasing metabolic demand, which in turn increases the demand on cardiac output. ${ }^{12}$ Every attempt should be made to optimize management of myocardial ischemia, fluid status, systolic and diastolic ventricular function, and control of arrhythmias. This approach can help reduce cardiac disease limiting the weaning process. In the cardiac surgery population, independent predictors of PMV include preoperative renal failure, postoperative stroke, intra-aortic balloon pump insertion, emergency operation, complete heart block, and longer perfusion time. ${ }^{13}$ Echocardiography and measurements of B-type natriuretic peptides can be very useful tools to help diagnose weaning failure of cardiac origin. ${ }^{12,14,15}$ Patients on PMV with automated implantable cardioverter defibrillator devices who have limited their code status or want to discontinue MV completely will require the defibrillator component of the device to be disabled to prevent unwanted cardioversion or pacing during compassionate weaning.

Impaired central drive will result in absent or severely diminished ventilatory activity during weaning trials. This is usually due to damage to the neurons controlling respiratory drive in the brainstem from cerebrovascular or post neurosurgical procedures. Central drive can also be reversibly inhibited with sedating medications (used to manage 
pain, anxiety, or delirium), metabolic alkalosis, or respiratory alkalosis. Resolution of metabolic complications of chronic critical illness and tapering of sedating medications can allow SBTs to commence.

Critical illness neuromyopathy (CINM) manifests itself as ICU acquired weakness, and its role in PMV has been recently reviewed in detail. ${ }^{16,17}$ The lack of both a clear definition of CINM and diagnostic criteria (eg, electrophysiological studies vs questionnaire) remain issues. Five risk factors for CINM have been identified from a number of recently performed prospective observational studies $^{18,19}$ : multiple organ failure, muscle inactivity, hyperglycemia, use of corticosteroids, and use of neuromuscular blockers. Effective preventive strategies are not well defined, but recent evidence suggests the following may help minimize CINM: early identification and treatment of severe sepsis and septic shock to reduce sepsis related myopathy, minimizing the use of deep sedation (role of antipsychotic use in the LTAC and weakness and PMV), avoidance of hyperglycemia, early mobilization (does not restore strength, but teaches how to handle weakness), and minimizing steroid use.

There is a broad differential diagnosis of respiratory muscle weakness in patients undergoing PMV, including preexisting conditions (such as Guillain-Barré syndrome, muscular dystrophy, hyperinflation, malnutrition, and thyroid dysfunction) and acquired conditions (such as CINM, electrolyte disorders, and medications such as steroids or aminoglycosides). ${ }^{20}$ Substantial respiratory muscle weakness has been shown to correlate with general muscle function and to be an independent predictor of time to wean from MV in the ICU. Thus, CINM likely involves the respiratory muscles and may affect time to wean from PMV in the post ICU setting. ${ }^{21}$

Ventilator-induced diaphragm dysfunction is a particularly rapid form of skeletal muscle injury, and has been shown to occur after only 18 hours of imposed diaphragm inactivity from MV. There is an extensive body of knowledge regarding the etiology of ventilator-induced diaphragm dysfunction. ${ }^{22}$ Oxidative stress, protein catabolism, reduced protein synthesis, and apoptosis are considered key causes. ${ }^{7,20}$ Data from animals also suggest that diaphragm inactivity may alter mitochondrial function and lead to increased production of reactive oxygen species. ${ }^{23}$ Age and continuous mandatory ventilation appear to have an additive negative effect on diaphragmatic function, which may explain the demographics of the population of patients undergoing PMV. Data from animal studies suggest that continuous mandatory ventilation per se may reduce the contractile properties of the diaphragm, through an effect on myofibrils. ${ }^{24}$ It has not been easy to isolate the effect of continuous mandatory ventilation from other potentially detrimental effects on the diaphragm, such as PEEP and neuromuscular blockers. Data from animal stud- ies support the use of continuous mandatory ventilation modes ${ }^{25}$ and pressure support ${ }^{26}$ rather than continuous mandatory ventilation modes to minimize ventilator-induced diaphragm dysfunction.

Suboptimal patient-ventilator synchrony can be an under-recognized cause of increased work of breathing, and has been extensively reviewed. ${ }^{10,27}$ Achieving optimal patient-ventilator synchrony during PMV depends on a number of factors, including adjusting ventilator settings in response to improving lung compliance and inspiratory muscle strength, adjusting psychotropic medications to optimize mental status, and ensuring optimal tracheostomy tube function. Optimal patient-ventilator synchrony implies that every breath initiated by the patient is matched by the ventilator during the respiratory cycle. A mismatch between the patient's ventilatory requirements and the delivered machine breath can lead to either inadequate or excessive assistance from the mechanical ventilator. Inadequate ventilator assistance leads to insufficient unloading of the respiratory muscles. Providing excessive ventilator assistance increases the risk of worsening dynamic hyperinflation and auto-PEEP in patients with obstructive disease, and in addition may cause hypocapnia with resulting central apneas and arousals from sleep. An incorrectly set flow rate set can also increase work of breathing. Too high a setting can result in premature breath termination with double triggering, and too low can lead to patient discomfort and an inadequate expiratory time. Careful review of the ventilator settings along with the number of hours the patient needs long-term MV will help avoid these problems. The ability to interpret ventilator graphics, along with awareness of the causes of patient ventilator asynchrony, is an important skill in the post acute care setting, where many patients are weaned from PMV.

\section{Special Situations}

There have been important advances in the care of certain patient populations with pulmonary disease over the past 2 decades. Lung transplantation, new therapeutic options for pulmonary hypertension, and diaphragm pacing in particular have growing relevance to patients undergoing PMV.

A small but growing number of patients with interstitial lung disease, cystic fibrosis, and COPD undergoing PMV may be candidates for lung transplantation. The selection and preparation of a patient for lung transplant from PMV is both complex and rigorous, as outcomes for these patients can be worse compared with non-ventilated patients undergoing lung transplantation. ${ }^{28}$

Respiratory failure and PMV can occur as a complication of pulmonary hypertension (usually primary). The advances in treatment of pulmonary hypertension have resulted in improved survival in this population, ${ }^{29}$ and in some cases allowed successful weaning from PMV. 
Diaphragm pacing has been used to liberate patients with spinal cord injury from PMV. Diaphragm pacing has also been reported to delay the need for MV by up to 2 years in patients with amyotrophic lateral sclerosis. ${ }^{30,31}$

\section{Systematic Approach to Weaning Trials/ Liberation Trials}

Patients who require PMV have, by definition, failed multiple SBTs and required the placement of a tracheostomy tube. In the acute care setting a bundled approach has been proposed in an attempt to both shorten MV time and also optimize function following critical illness. For example, Banerjee et $\mathrm{al}^{32}$ have proposed the "ABCDE" bundle (attention to daily Awakening, spontaneous Breathing trials, sedation Choice, Delirium monitoring, and Exercise/early mobility) to improve functional and cognitive abilities of survivors of critical illness. The relevance of this type of approach to patients undergoing PMV is unclear, but accumulating data indicate that opportunities exist to improve the management of delirium and immobility in the LTAC setting at least. ${ }^{32}$

A summary of data available to date in 2005 was presented at the National Association of Medical Directors for Respiratory Care conference. ${ }^{3}$ At that conference, liberation from PMV for 7 consecutive days was, by consensus, considered to be weaning from PMV. The recommendations for weaning at the time included weaning the PMV patient to about $50 \%$ of ventilatory requirements using pressure support mode $\left(10-15 \mathrm{~cm} \mathrm{H}_{2} \mathrm{O}\right)$ and then instituting therapist driven SBTs of increasing duration via a tracheostomy mask or T-piece. This approach can shorten the time to weaning by about 12 days in the PMV population. ${ }^{33}$ A rapid shallow breathing index of up to 97 has been shown to correlate with successful 1-hour tolerance of an SBT in the PMV population. ${ }^{34}$ There is some evidence that systematic SBTs done at the time of admission to the post acute setting may rapidly identify a subset of patients with COPD who can start to wean quickly from PMV. ${ }^{35}$ In a review of LTACs in 2006, up to $70 \%$ reported using a non-physician-driven protocol (National Association of Long Term Hospital 2011 Physician Clinical Education Conference: Long Term Acute Care: Turning Knowledge Into Practice, October 6-7, 2011, New Orleans, Louisiana).

Has anything changed over the past 6 years? Robust predictors of successful weaning in the PMV population have been difficult to identify. A recent Cochrane review of 11 trials concluded that there are not enough data to unequivocally support the use of weaning protocols in the ICU setting. ${ }^{36}$ There are some data suggesting that noninvasive ventilation (NIV) can be used to shorten time to extubation in the ICU and significantly reduce mortality and ventilator-associated pneumonia rates, ${ }^{37}$ but it is not known if NIV provides any similar benefit in the PMV
Table 1. Outcome* of Patients Admitted to Long-Term Acute Care Hospitals on Prolonged Mechanical Ventilation

\begin{tabular}{lr}
\hline \hline \multicolumn{1}{c}{ Outcome } & $\%$ \\
\hline Weaned and decannulated & 31 \\
Weaned not decannulated & 22 \\
Full time mechanical ventilation via tracheostomy & 16 \\
Decannulated, required noninvasive mechanical ventilation & 6 \\
Died & 25 \\
& \\
* Data collected at the time of discharge. & \\
(Data from Reference 41.) & \\
\hline
\end{tabular}

population. The recovery of inspiratory muscle force has been found to be a major determinant of "late" weaning success, by allowing the patient undergoing PMV to breathe substantially below the diaphragm fatigue threshold. ${ }^{38}$ There are several approaches to wean patients from PMV, including a gradual reduction of pressure support ventilation, SBTs, and capping of the tracheostomy tube with NIV. Data obtained in the 1990s in patients undergoing short-term MV $(<21 \mathrm{~d})$ demonstrate that synchronized intermittent mandatory ventilation (SIMV) as a weaning mode is inferior to T-piece or pressure support ventilation. ${ }^{39,40}$ A protocol that combined SIMV, pressure support ventilation, and SBTs in a step-wise manner was effective in reducing weaning time in patients weaning from PMV at an LTAC. ${ }^{33}$ In the most comprehensive study to date of outcomes in patients referred to LTACs for weaning from PMV no data on weaning ${ }^{41}$ mode were included. A recent study used a tracheostomy mask weaning protocol exclusively to wean patients from PMV. ${ }^{42}$

Weaning rates in the PMV population have varied significantly (range $42-83 \%$, average $54 \%$ ); this variation is probably related to the heterogeneity of the population of patients who undergo PMV. ${ }^{11,34,41}$ Outcome data from the ventilator outcomes study performed in 23 LTACs are summarized in Table $1 . .^{41}$ The use of weaning protocols in the ICU setting has helped standardize the weaning process and has resulted in a reduction in the number of days to extubation, decreased the need for tracheostomy, and lowered ICU costs. ${ }^{2}$ Weaning protocols have also been successfully employed to reduce weaning times in PMV patients at LTAC hospitals ${ }^{43}$ and weaning units in acute care facilities. ${ }^{44}$ In reality, the variable recovery trajectories of patients undergoing PMV may be impossible to synchronize with any weaning protocol, and an individualized multidisciplinary approach is likely the most effective strategy. Better identification of subsets of patients undergoing PMV is needed to individualize both treatment and weaning strategies. It is unclear how useful novel strategies such as weaning during bathing ${ }^{45}$ will be over time. 


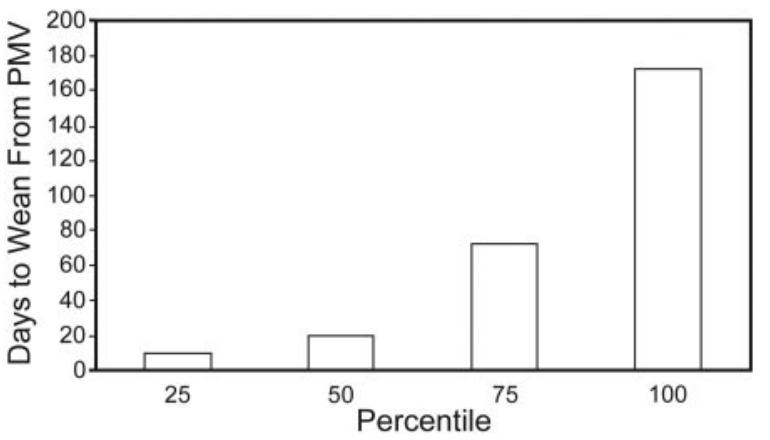

Fig. 1. Days to wean from prolonged mechanical ventilation following admission to a long-term acute care hospital. Data are presented in quartiles.

\section{Failure to Wean and the Decision to Proceed With Life-Long Support}

A study carried out in 23 LTACs in the United States remains an important source of weaning data on the PMV population admitted to LTACs. ${ }^{11,41}$ The data obtained in this prospective observational cohort study of 1,419 patients yielded key metrics on failure to wean from PMV (see Table 1). In this study, $20 \%$ of patients undergoing PMV in the LTAC setting failed to wean from PMV. Of those failing to wean, almost $80 \%$ required full time PMV, $18 \%$ required part time PMV, and $2 \%$ were managed with NIV. The median number of days to wean from PMV was 15 days. In 3 of the 23 LTACs the 75th percentile of days to wean was 46 days. The remaining LTACs had lower values for this metric. Thus, if a patient has not weaned by day 46 in an LTAC, they may have a high likelihood of remaining ventilator dependent. Similar data were obtained in a single center United Kingdom based study. ${ }^{46}$ Unpublished data from our ventilator database for 2010 are shown in Figure 1. During this time period $60 \%$ of 110 patients admitted for management of PMV were successfully weaned. The time to weaning to tracheostomy mask following LTAC admission is shown in quartiles. The median time to wean was 19 days (50th percentile), with $75 \%$ of patients weaning by day 72 . Failure to wean from PMV, resulting in the need for long-term $\mathrm{MV}$, occurred in $66 \%$ of patients with neuromuscular disease, $20 \%$ of those with COPD, and $14 \%$ of those with a surgical cause for PMV. ${ }^{46}$ The relatively high weaning rates for COPD patients reported in this study $(>50 \%)$ may relate to a more restrictive ICU admission practice for patients with COPD in the United Kingdom, as compared with the United States. In addition, almost $40 \%$ of the patients included in this study did not meet the criteria for PMV, as they had not undergone MV for $>21$ days at the time of admission to the facility.

Some patients undergoing PMV and recovering from chronic critical illness have a prolonged weaning and re- covery trajectory. In general, the probability of a patient weaning from PMV at any point in time appears to be influenced by the little understood "repair/recovery process" and also by the number of days of PMV elapsed to date. Increasing age, stay in the referring ICU, and severity of illness as measured by Acute Physiology and Chronic Health Evaluation II score have been associated with worse outcomes. ${ }^{46}$ Failure to wean from PMV has also been linked to an elevated body mass index ${ }^{42}$ increased stay in the weaning unit, elevated blood urea nitrogen levels, lower modified Glasgow coma scale, serum albumin, and reduced maximal inspiratory pressure. ${ }^{47}$ In general, a multidisciplinary approach to care that includes the systematic treatment of barriers to weaning, as noted above, along with careful attention to other details of medical care, such as glucose control, ${ }^{48}$ is needed to optimize each patient's chances of weaning from PMV. Despite the need for longterm MV, lung compliance and respiratory muscle strength should be assessed regularly, until the patient has reached a functional plateau. Any improvement in these parameters may allow the patient to wean for a few hours a day, which can substantially improve quality of life by enhancing communication and a sense of independence.

The decision to transition from PMV to long-term MV and abandon any hope of weaning will be driven by patient and family preferences, which ideally are aligned with the clinical reality. Patients with isolated respiratory failure from COPD or neuromuscular disease are more amenable to long-term MV. Patients with other comorbidities, such as non-healing decubitus ulcers or renal failure requiring hemodialysis, have a more uncertain shortterm and long-term outcome. ${ }^{1}$ Patients can undergo longterm MV in a number of settings, including home, skilled nursing facility, or LTAC.

Helping patients and families understand the concept of futile treatment and then move toward palliative care is not easy. Recovery and weaning expectations are set early in the course of critical illness, and frequently result in tracheostomy placement and a decision to pursue PMV. Resetting expectations in the setting of recovery and weaning failure can be very difficult for some patients and families. Recent data obtained in a respiratory unit indicate the need for better communication between caregivers, patients, and families about decisions concerning PMV. ${ }^{49}$ Deficiencies in palliative care delivery have been identified in the ICU setting, which in turn drive unrealistic expectations for PMV. Improving caregiver knowledge about palliative care, enhancing "spiritual" support for families, and providing emotional support for clinicians, such as "Schwartz rounds," ${ }^{50}$ are areas of identified need in the ICU setting. More work is needed to determine if similar deficits are present in the LTAC setting. Compassionate weaning from PMV (removing the patient from the mechanical ventilator and treating for comfort) can be performed successfully in 
the LTAC setting, and differs from that performed in the ICU in a number of ways. ${ }^{51}$

Outcome data show substantial mortality over time for subsets of patients who require long-term MV. The 3 year survival rate is $<50 \%$ for patients with COPD, and about $70 \%$ for patients with either neuromuscular disease or who are post surgery. ${ }^{46}$ Patients who require PMV who are $>65$ years of age and have sacral ulcers and abnormal renal function appear to have poor long-term survival. ${ }^{52}$ More research is needed to better identify treatments to enhance weaning, improve skin integrity and preserve or improve renal function. It is important for clinicians who care for patients undergoing long-term MV to continually reassess the patient's goals and to clearly identify advance directives and, if possible, to establish healthcare proxies before a patient loses the ability to communicate.

\section{Tracheostomy Tube Management During PMV}

A correctly sized and positioned tracheostomy tube is essential for invasive (as opposed to noninvasive) PMV. The placement of a tracheostomy tube allows the removal of a translaryngeal endotracheal tube and allows the safe transfer of the patient from the ICU to a lower level of care, such as a weaning unit. A tracheostomy tube has a number of advantages over an endotracheal tube. These include the prevention of further damage to the posterior aspect of the vocal cords, ${ }^{53}$ the reduction in the work of breathing, ${ }^{54}$ the reduction in sedation and analgesia, improved oral care, and, finally, the recovery of speech and swallowing.

A tracheostomy tube is placed using either a surgical or percutaneous approach. ${ }^{55}$ The typical tube inserted at the time of tracheostomy is made of polyvinyl chloride and has an air filled cuff. A tube with an inner diameter of at least $7.5 \mathrm{~mm}$ may be required initially in some patients, to allow the passage of a bronchoscope. Because of the risks associated with accidental decannulation (AD) of a freshly placed tracheostomy tube, most surgeons secure the tube to the neck by suturing the flange to the skin. ${ }^{56}$ In addition, stay sutures ${ }^{57}$ may be placed to allow easy access to the immature tracheocutaneous fistula, in the event of an AD. These sutures can be life-saving in an AD in the obese patient with increased neck circumference. The stay sutures can be removed at the time of the first tracheostomy tube change.

The first tracheostomy tube change can be safely done once the tracheotomy stoma tract has matured, and by convention is performed 5-7 days after placement. ${ }^{58}$ The first tube change may be done in either a weaning unit or an LTAC, provided the necessary skills and equipment are available. The first tube change facilitates the removal of retaining sutures, which can be uncomfortable for the patient. This topic has been recently reviewed. ${ }^{59} \mathrm{~A}$ smaller fiberoptic tracheoscope can be passed through tracheos- tomy tubes as small as $5 \mathrm{~mm}$ inner diameter, but due to their small diameter these endoscopes do not have suction capability. The risk of airway loss during the first tube change can be reduced by having a skilled operator make the first tube change and, if necessary, a simple tube changer can be used. ${ }^{59}$ Airway loss during a tracheostomy tube change can be catastrophic and may result in the patient requiring translaryngeal reintubation.

Selecting the correct tracheostomy tube depends on a number of variables. The female trachea is smaller than the male trachea, due to the inhibitory effect of menarche on tracheal and other tissue growth trajectories during adolescence. A size 6-6.5 mm inner diameter tube is usually adequate for females, with males needing a tube that is $1 \mathrm{~mm}$ larger (7-7.5 mm inner diameter). The tube selected should be long enough to ensure the distal end of the tracheostomy tube is centrally placed in the airway. In general, larger tracheostomy tubes should be down-sized to these sizes to minimize trauma to the tracheal wall, to prevent the formation of granulation tissue, and to enhance speech by allowing air flow to the vocal cords when the cuff is deflated. An additional benefit of down-sizing the tube is to expedite healing of the tracheostomy stoma after planned decannulation.

A tube that is either too long or too short may lie against the anterior or posterior tracheal wall and result in patientventilator asynchrony. A malpositioned tube in the airway can be partially occluded and may increase the work of breathing, prolong the duration of MV by interfering with weaning from PMV, interfere with effective suctioning, and result in a rise in the peak airway pressure needed to deliver the set tidal volume. ${ }^{60,61}$ Transitioning a patient to tracheostomy mask from positive pressure MV can unmask tube malposition, as the dilating effect of the positive pressure is eliminated during the wean. ${ }^{61}$ The causes of malposition have been recently reported and include occlusion of the tube by the anterior or posterior wall of the trachea or granulation tissue. A tracheostomy tube of insufficient or excessive length may also abut adjacent tissues and occlude. A tracheoscope can be an invaluable aid to ensuring the tracheostomy tube is correctly positioned within the tracheal lumen.

If the patient has started SBTs using a tracheostomy mask, the insertion of a silicone tube with a low profile water filled cuff (as compared with the more bulky air filled cuff) may further facilitate speech by improving air flow through the subglottic space and over the vocal cords. An inflated cuff is essential to allow for MV. The cuff is deflated during tracheostomy mask weaning trials and also to allow for leak speech in selected patients during MV. Tracheostomy tubes with inner cannulas are not useful in patients weaning from PMV, unless there are issues with mucus plugging necessitating frequent exchange of the inner cannula to maintain patency of the tracheostomy 
tube. Fenestrated tubes in theory are useful to augment speech, but, in reality, ensuring the fenestrations are correctly positioned within the airway lumen can be challenging, and fenestrations may promote the formation of granulation tissue on the tracheal wall if the tube is not correctly placed. ${ }^{62}$ In patients with substantial tracheomalacia or granulation tissue formation, a tube with an adjustable flange may be helpful, by allowing the tube length to be adjusted to ensure the distal end of the tracheostomy tube remains clear of debris over a prolonged period of time.

There are limited data available and no guidelines to help determine when to change a tracheostomy tube in adults after the first change has been made. ${ }^{59}$ Tracheostomy tubes need to be changed prior to device fatigue, to comply with manufacturers' guidelines and also to minimize the risk of occlusion due to biofilm buildup. ${ }^{63}$ Based on our review of the available literature and manufacturers' guidelines, we recommend maximal time periods of about 2-3 months between tracheostomy tube changes. In some chronically critically ill patients with excessive secretions due to multiple-drug-resistant healthcare associated pneumonias or end stage cardiac disease, more frequent changes may be needed. In some instances, additional measures to reduce secretions are worth attempting, such as scopolamine patches ${ }^{64}$ or parotid gland injection with botulinum toxin. ${ }^{65}$

Once patients start to tolerate SBTs, a one way speaking valve $^{66}$ can be used to allow phonation over the vocal cords and also enhance swallowing by augmenting subglottic pressure. In patients who cannot wean from MV these devices can theoretically be used "in line" to allow speech in mechanically ventilated patients. However, this must be done with extreme caution, as the in-line use of speaking valves requires deflating the cuff and disabling the expiratory volume alarm on the mechanical ventilator. Carefully selected patients with neuromuscular disease with minimal secretions may tolerate this approach, which allows for better communication. An alternative approach is volume compensation "leak speech." 67

Complete occlusion of the tracheostomy tube with a cap is the final step before planned decannulation. Substantial glottic or subglottic pathology will usually become apparent during capping trials. Tolerance of capping is essential to planned decannulation. Overall about $35 \%$ of patients referred to LTACs for PMV are successfully decannulated. 68

Accidental decannulation, like accidental extubation, ${ }^{69}$ can be life threatening and has not been well studied to date. Data on the causes of AD at our facility have been recently submitted for publication. Factors associated with AD in the LTAC setting included mental status changes (including delirium), poorly secured tracheostomy ties, and excessive secretions. Reducing the number of $\mathrm{AD}$ events should be an important quality goal for weaning units.

\section{How Does One Select a Ventilator for the LTAC, Compared to the ICU?}

The MV needs of patients admitted to a weaning or step-down unit should be stable, and ideally less than those required in the ICU. The only exception to this general rule is the small subset of patients who are declining continued aggressive care and have limitations on their code status and do not want to be readmitted to the ICU.

Patients who require PMV are usually managed using volume-controlled continuous mandatory ventilation with a set tidal volume of $8 \mathrm{~mL} / \mathrm{kg}$ (giving a tidal volume of about $400-600 \mathrm{~mL}$ ), $\mathrm{F}_{\mathrm{IO}_{2}}$ of $<50 \%$, and a PEEP of $<8 \mathrm{~cm} \mathrm{H}_{2} \mathrm{O}$. Many patients recovering from either acute lung injury or some risk factor for acute lung injury need PMV. These patients are typically receiving a tidal volume of $6 \mathrm{~mL} / \mathrm{kg}$, based on the ARDS Network data. ${ }^{70}$ With improving lung compliance and increasing function capacity, higher tidal volumes may be needed to prevent atelectasis and the need for increased $\mathrm{F}_{\mathrm{IO}_{2}}$ and PEEP. ${ }^{71}$ Other modes are sometimes used, such as SIMV, pressure-controlled continuous mandatory ventilation, or pressure support ventilation. Volume-controlled continuous mandatory ventilation has been shown to minimize sleep fragmentation from central apneas, compared with pressure support ventilation..$^{72}$

The use of ventilator graphics allows the practitioner to easily visualize the airway pressure waveforms and determine if the patient is making too little or too much effort during each respiratory cycle. Graphic displays also allow one to visualize the flow-volume loop in patients with obstructive lung disease and pressure volume curves in patients with interstitial disease. This allows optimal patient-ventilator synchrony and facilitates weaning. A further discussion of patient-ventilator synchrony in the patient who requires PMV has been previously published. ${ }^{10}$

Therefore, the ideal ventilator to use in the patient who needs PMV in the LTAC setting should have a graphic display, have a selection of basic modes of MV available (such as continuous mandatory ventilation and pressure support), and be able to calculate static, dynamic compliance, and auto-PEEP. Weaning trials are essential to initial patient management, so having a highly responsive inspiratory flow system is important to minimize the work of breathing, along with precise exhaled tidal volume monitoring capability. With the increasing use of NIV beyond the ICU, it is advantageous if the mechanical ventilator can also be used to deliver NIV. Dedicated NIV devices are also used in greater numbers in the LTAC setting for this reason. The recent evidence supporting the use of early mobility during $\mathrm{MV}^{73,74}$ supports the use of mechanical ventilators that can be moved around easily with the patient. Hence portability (weight, mobility, internal bat- 
tery life) are also important factors to keep in mind when selecting mechanical ventilators for the LTAC setting.

\section{Summary}

Strategies to effectively manage patients who require long term MV include systematically addressing barriers to weaning, ensuring appropriate weaning trials, optimizing the tracheostomy tube, and setting a realistic time frame for weaning attempts.

\section{REFERENCES}

1. Nelson JE, Cox CE, Hope AA, Carson SS. Chronic critical illness. Am J Respir Crit Care Med 2010;182(4):446-454.

2. Boles JM, Bion J, Connors A, Herridge M, Marsh B, Melot C, et al. Weaning from mechanical ventilation. Eur Respir J 2007;(5):10331056.

3. MacIntyre NR, Epstein SK, Carson S, Scheinhorn D, Christopher K, Muldoon S, et al. Management of patients requiring prolonged mechanical ventilation: report of a NAMDRC consensus conference. Chest 2005;128(6):3937-3954.

4. Farfel JM, Franca SA, Sitta MdC, Filho WJ, Carvalho CRR. Age, invasive ventilatory support and outcomes in elderly patients admitted to intensive care units. Age Ageing 2009;(5):515-520.

5. Topeli A, Cakir B. Hospital survival of older patients admitted to a medical intensive care unit. Aging Clin Exp Res 2007;(1):69-74.

6. Criswell DS, Shanely RA, Betters JJ, McKenzie MJ, Sellman JE, Van Gammeren DL, et al. Cumulative effects of aging and mechanical ventilation on in vitro diaphragm function. Chest 2003;(6):23022308.

7. Levine S, Nguyen T, Taylor N, Friscia ME, Budak MT, Rothenberg $\mathrm{P}$, et al. Rapid disuse atrophy of diaphragm fibers in mechanically ventilated humans. N Engl J Med 2008;358(13):1327-1335.

8. Rennie MJ. Anabolic resistance in critically ill patients. Crit Care Med 2009;37(10 Suppl):S398-S399.

9. Pisani MA. Considerations in caring for the critically ill older patient. J Intensive Care Med 2009;24(2):83-95.

10. Ghamloush M, O'Connor HH, White AC. Patient-ventilator interaction in the long-term acute-care hospital. Respir Care 2011;56(2): 207-213.

11. Scheinhorn D, Hassenpflug M, Votto J, Chao D, Epstein SK, Doig $\mathrm{G}$, et al. Ventilator-dependent survivors of catastrophic illness transferred to 23 long term care hospitals for weaning from prolonged mechanical ventilation. Chest 2007;131(1):76-84.

12. Grasso S, Leone A, De Michele M, Anaclerio R, Cafarelli A, Ancona $\mathrm{G}$, et al. Use of N-terminal pro-brain natriuretic peptide to detect acute cardiac dysfunction during weaning failure in difficult-to-wean patients with chronic obstructive pulmonary disease. Crit Care Med 2007;35(1):96-105.

13. Shirzad M, Karimi A, Ahmadi SH, Marzban M, Tazik M, Aramin H. Predictors and early outcome of prolonged mechanical ventilation in contemporary heart valve surgery. Monaldi Arch Chest Dis 2010; 74(1):22-27.

14. Zapata L, Vera P, Roglan A, Gich I, Ordonez-Llanos J, Betbese AJ. B-type natriuretic peptides for prediction and diagnosis of weaning failure from cardiac origin. Intensive Care Med 2011;37(3):477-485.

15. Adamopoulos C, Tsagourias M, Arvaniti K, Veroniki F, Matamis D. Weaning failure from mechanical ventilation due to hypertrophic obstructive cardiomyopathy. Intensive Care Med 2005;31(5):734-737.

16. de Jonghe B, Lacherade J-C, Sharshar T, Outin H. Intensive care unit-acquired weakness: risk factors and prevention. Crit Care Med 2009;37(10 Suppl):S309-S315.
17. Fan E, Zanni JM, Dennison CR, Lepre SJ, Needham DM. Critical illness neuromyopathy and muscle weakness in patients in the intensive care unit. AACN Adv Crit Care 2009;20(3):243-253.

18. Sharshar T, Bastuji-Garin S, De Jonghe B, Stevens RD, Polito A, Maxime V, et al. Hormonal status and ICU-acquired paresis in critically ill patients. Intensive Care Med 2010;36(8):1318-1326.

19. Stevens RD, Dowdy DW, Michaels RK, Mendez-Tellez PA, Pronovost PJ, Needham DM. Neuromuscular dysfunction acquired in critical illness: a systematic review. Intensive Care Med 2007;33(11): 1876-1891.

20. Tobin MJ, Laghi F, Jubran A. Narrative review: ventilator-induced respiratory muscle weakness. Ann Intern Med 2010;153(4):240-245.

21. De Jonghe B, Bastuji-Garin S, Durand M-C, Malissin I, Rodrigues P, Cerf C, et al. Respiratory weakness is associated with limb weakness and delayed weaning in critical illness. Crit Care Med 2007;35(9): 2007-2015.

22. Haitsma JJ. Diaphragmatic dysfunction in mechanical ventilation. Curr Opin Anaesthesiol 2011;24(2):214-218.

23. Kavazis AN, Talbert EE, Smuder AJ, Hudson MB, Nelson WB, Powers SK. Mechanical ventilation induces diaphragmatic mitochondrial dysfunction and increased oxidant production. Free Radic Biol Med 2009;46(6):842-850.

24. Sassoon CS, Caiozzo VJ. Bench-to-bedside review: diaphragm muscle function in disuse and acute high-dose corticosteroid treatment. Crit Care 2009;13(5):221.

25. Sassoon CSH, Zhu E, Caiozzo VJ. Assist-control mechanical ventilation attenuates ventilator-induced diaphragmatic dysfunction. Am J Respir Crit Care Med 2004;170(6):626-632.

26. Futier E, Constantin J-M, Combaret L, Mosoni L, Roszyk L, Sapin $\mathrm{V}$, et al. Pressure support ventilation attenuates ventilator-induced protein modifications in the diaphragm. Crit Care 2008;12(5):R116.

27. Epstein SK. How often does patient-ventilator asynchrony occur and what are the consequences? Respir Care 2011;56(1):25-38.

28. Elizur A, Sweet SC, Huddleston CB, Gandhi SK, Boslaugh SE, Kuklinski CA, et al. Pre-transplant mechanical ventilation increases short-term morbidity and mortality in pediatric patients with cystic fibrosis. J Heart Lung Transplant 2007;26(2):127-131.

29. Driscoll JA, Chakinala MM. Medical therapy for pulmonary arterial hypertension. Expert Opin Pharmacother 2008;9(1):65-81.

30. Onders R, McGee MF, Marks J, Chak A, Schilz R, Rosen MJ, et al. Diaphragm pacing with natural orifice transluminal endoscopic surgery: potential for difficult-to-wean intensive care unit patients. Surg Endosc 2007;21(3):475-479.

31. Onders RP, Elmo M, Khansarinia S, Bowman B, Yee J, Road J, et al. Complete worldwide operative experience in laparoscopic diaphragm pacing: results and differences in spinal cord injured patients and amyotrophic lateral sclerosis patients. Surg Endosc 2009;23(7): 1433-1440.

32. Banerjee A, Girard TD, Pandharipande P. The complex interplay between delirium, sedation, and early mobility during critical illness: applications in the trauma unit. Curr Opin Anaesthesiol 2011;24(2): 195-201.

33. Scheinhorn DJ, Chao DC, Stearn-Hassenpflug M, Wallace WA. Outcomes in post-ICU mechanical ventilation: a therapist-implemented weaning protocol. Chest 2001;119(1):236-242.

34. Chao DC, Scheinhorn DJ. Determining the best threshold of rapid shallow breathing index in a therapist-implemented patient-specific weaning protocol. Respir Care 2007;52(2):159-165.

35. Vitacca M, Vianello A, Colombo D, Clini E, Porta R, Bianchi L, et al. Comparison of two methods for weaning patients with chronic obstructive pulmonary disease requiring mechanical ventilation for more than 15 days. Am J Respir Crit Care Med 2001;164(2):225-230.

36. Blackwood B, Alderdice F, Burns K, Cardwell C, Lavery G, O'Halloran P. Use of weaning protocols for reducing duration of 
mechanical ventilation in critically ill adult patients: Cochrane systematic review and meta-analysis. BMJ 2011;342:c7237.

37. Burns KE, Adhikari NK, Keenan SP, Meade MO. Noninvasive positive pressure ventilation as a weaning strategy for intubated adults with respiratory failure. Cochrane Database Syst Rev 2010(8):CD004127.

38. Carlucci A, Ceriana P, Prinianakis G, Fanfulla F, Colombo R, Nava $\mathrm{S}$. Determinants of weaning success in patients with prolonged mechanical ventilation. Crit Care 2009;13(3):R97.

39. Brochard L, Rauss A, Benito S, Conti G, Mancebo J, Rekik N, et al. Comparison of three methods of gradual withdrawal from ventilatory support during weaning from mechanical ventilation. Am J Respir Crit Care Med 1994;150(4):896-903.

40. Esteban A, Frutos F, Tobin MJ, Alia I, Solsona JF, Valverdu I, et al. A comparison of four methods of weaning patients from mechanical ventilation. Spanish Lung Failure Collaborative Group. N Engl J Med 1995;332(6):345-350.

41. Scheinhorn D, Hassenpflug M, Votto J, Chao D, Epstein SK, Doig $\mathrm{G}$, et al. Post-ICU mechanical ventilation at 23 long-term care hospitals: a multicenter outcomes study. Chest 2007;131(1):85-93.

42. Mamary AJ, Kondapaneni S, Vance GB, Gaughan JP, Ubado JM, Criner GJ. Survival in patients receiving prolonged mechanical ventilation: Factors that influence outcome Clin Med Insights 2011;5: 17-26.

43. Scheinhorn DJ, Chao DC, Hassenpflug MS, Gracey DR, Scheinhorn DJ, Chao DC, et al. Post-ICU weaning from mechanical ventilation: the role of long-term facilities. Chest 2001;120(6 Suppl):482S-484S.

44. Bigatello LM, Stelfox HT, Berra L, Schmidt U, Gettings EM. Outcome of patients undergoing prolonged mechanical ventilation after critical illness. Crit Care Med 2007;35(11):2491-2497.

45. Sereika SM, Tate JA, DiVirgilio-Thomas D, Hoffman LA, Swigart VA, Broyles L, et al. The association between bathing and weaning trial duration. Heart Lung 2011;40(1):41-48.

46. Pilcher DV, Bailey MJ, Treacher DF, Hamid S, Williams AJ, Davidson AC. Outcomes, cost and long term survival of patients referred to a regional weaning centre. Thorax 2005;60(3):187-192.

47. Wu Y-K, Kao K-C, Hsu K-H, Hsieh M-J, Tsai Y-H. Predictors of successful weaning from prolonged mechanical ventilation in Taiwan. Respir Med 2009;103(8):1189-1195.

48. Wieske L, Harmsen RE, Schultz MJ, Horn J. Is critical illness neuromyopathy and duration of mechanical ventilation decreased by strict glucose control? Neurocritical Care 2011;14(3):475-481.

49. Camhi SL, Mercado AF, Morrison RS, Du Q, Platt DM, August GI, et al. Deciding in the dark: advance directives and continuation of treatment in chronic critical illness. Crit Care Med 2009;37(3):919-925.

50. Penson RT, Schapira L, Mack S, Stanzler M, Lynch TJ Jr. Connection: Schwartz Center Rounds at Massachusetts General Hospital Cancer Center. Oncologist 2010;15(7):760-764.

51. White AC, Joseph B, Gireesh A, Shantilal P, Garpestad E, Hill NS, et al. Terminal withdrawal of mechanical ventilation at a long-term acute care hospital: comparison with a medical ICU. Chest 2009; 136(2):465-470.

52. Aboussouan LS, Lattin CD, Kline JL. Determinants of long-term mortality after prolonged mechanical ventilation. Lung 2008;186(5): 299-306.

53. Weber S. Traumatic complications of airway management. Anesthesiol Clin North America 2002;20(3):503-512.

54. Diehl JL, El Atrous S, Touchard D, Lemaire F, Brochard L. Changes in the work of breathing induced by tracheotomy in ventilator-dependent patients. Am J Respir Crit Care Med 1999; 159(2):383-388.

55. Engels PT, Bagshaw SM, Meier M, Brindley PG. Tracheostomy: from insertion to decannulation. Can J Surg 2009;52(5):427-433.

56. Liew L, Gibbins N, Oyarzabal M. How I do it: securing tracheostomy tubes. Eur Arch Otorhinolaryngol 2008;265(5):607-608.
57. Burke A. The advantages of stay sutures with tracheostomy Ann Royal Coll Surg Engl 1981;63(6):426-428

58. Tabaee A, Lando T, Rickert S, Stewart MG, Kuhel WI, Tabaee A, et al. Practice patterns, safety, and rationale for tracheostomy tube changes: a survey of otolaryngology training programs. Laryngoscope 2007; 117(4):573-576.

59. White AC, Kher S, O'Connor HH. When to change a tracheostomy tube. Respir Care 2010;55(8):1069-1075.

60. Rumbak MJ, Walsh FW, Anderson WM, Rolfe MW, Solomon DA. Significant tracheal obstruction causing failure to wean in patients requiring prolonged mechanical ventilation: a forgotten complication of long-term mechanical ventilation. Chest 1999;115(4):10921095.

61. Schmidt U, Hess D, Kwo J, Lagambina S, Gettings E, Khandwala F, et al. Tracheostomy tube malposition in patients admitted to a respiratory acute care unit following prolonged ventilation. Chest 2008; 134(2):288-294.

62. Siddharth P, Mazzarella L. Granuloma associated with fenestrated tracheostomy tubes. Am J Surg 1985;150(2):279-280.

63. Backman S, Bjorling G, Johansson UB, Lysdahl M, Markstrom A, Schedin U, et al. Material wear of polymeric tracheostomy tubes: a six-month study. Laryngoscope 2009;119(4):657-664.

64. Tassinari D, Poggi B, Fantini M, Tamburini E, Sartori S. Treating sialorrhea with transdermal scopolamine. Exploiting a side effect to treat an uncommon symptom in cancer patients. Support Care Cancer 2005;13(7):559-561.

65. Young CA, Ellis C, Johnson J, Sathasivam S, Pih N. Treatment for sialorrhea (excessive saliva) in people with motor neuron disease/ amyotrophic lateral sclerosis. Cochrane Database Syst Rev 2011(5): CD006981.

66. Ohmae Y, Adachi Z, Isoda Y, Maekawa H, Kitagawa Y, Karaho T, et al. [Effects of one-way speaking valve placement on swallowing physiology for tracheostomized patients: impact on laryngeal clearance.] Nihon Jibinkoka Gakkai Kaiho 2006;109(7):594-599. Article in Japanese.

67. Hess DR. Facilitating speech in the patient with a tracheostomy. Respir Care 2005;50(4):519-525.

68. O'Connor HH, Kirby KJ, Terrin N, Hill NS, White AC. Decannulation following tracheostomy for prolonged mechanical ventilation. J Intensive Care Med 2009;24(3):187-194.

69. de Lassence A, Alberti C, Azoulay E, Le Miere E, Cheval C, Vincent $\mathrm{F}$, et al. Impact of unplanned extubation and reintubation after weaning on nosocomial pneumonia risk in the intensive care unit: a prospective multicenter study. Anesthesiology 2002;97(1): 148-156.

70. Eisner MD, Thompson T, Hudson LD, Luce JM, Hayden D, Schoenfeld D, et al. Efficacy of low tidal volume ventilation in patients with different clinical risk factors for acute lung injury and the acute respiratory distress syndrome. Am J Respir Crit Care Med 2001; 164(2):231-236.

71. Hager DN, Krishnan JA, Hayden DL, Brower RG, Network ACT. Tidal volume reduction in patients with acute lung injury when plateau pressures are not high. Am J Respir Crit Care Med 2005;172(10): 1241-1245.

72. Parthasarathy S. Sleep during mechanical ventilation. Curr Opin Pulm Med 2004;10(6):489-494.

73. Bailey PP, Miller RR 3rd, Clemmer TP. Culture of early mobility in mechanically ventilated patients. Crit Care Med 2009;37(10 Suppl): S429-S435.

74. Truong AD, Fan E, Brower RG, Needham DM. Bench-to-bedside review: mobilizing patients in the intensive care unit-from pathophysiology to clinical trials. Crit Care 2009;13(4):216. 


\section{Long-Term Mechanical Ventilation: Management Strategies}

\section{Discussion}

MacIntyre: In the long-term ventilated patient, what's the difference for someone with a tracheostomy with a pressure support of $10 \mathrm{~cm} \mathrm{H}_{2} \mathrm{O}$ versus NIV via face mask?

White: I think it probably doesn't matter whether you're getting NIV via face mask or nasal pillows or pressure support through a tracheostomy for a period of time in the LTAC, but when you're trying to get the patient home and to figure out what their care needs will be, its easier if you can eliminate suctioning and tracheostomy changes and all the equipment that goes along with a tracheostomy. The problem is that decannulation and moving to NIV can place some patients at risk. You have to select those patients very carefully.

MacIntyre: In the acute care setting there's this huge drive to do SBTs in recovering patients, to understand when somebody is ready to come off the ventilator. Usually by the time they've gotten to a long-term facility, however, they've had many failed SBTs. So at what point should you abandon SBTs, and then when do you restart them in the long-term setting? At a consensus conference several years ago, several people suggested that, instead of trying to do daily SBTs immediately in an LTAC patient, try to bring the ventilator support down a substantial amount. The question is, what's a substantial amount? At what level of pressure support or IMV or whatever should you start reconsidering SBTs?

White: I think pressure support of $7 / 5$ with adequate minute ventilation and the patient comfortable on that for one to two hours.

Hess: To follow up on that, how much of liberating the patient from the ventilator is related to the weaning strategy and how much is related to fixing the underlying disease processes? Because if a lot of it is fixing the underlying disease processes, then I would argue we should focus on that and do SBTs like we do in the acute care setting.

White: Yes, I read your editorial ${ }^{1}$ on that and I think you're absolutely right. Some physicians disagree that weaning actually does anything, and really all you're doing is waiting for the barriers to weaning to improve. I think that's true. We're basically waiting for the patient to be ready to wean, and an SBT essentially asks them if they're ready. We test their physiology. And what you do with the ventilator prior to that is probably unimportant; I think we're treating ourselves.

1. Hess DR, MacIntyre NR. Ventilator discontinuation: why are we still weaning? Am J Respir Crit Care Med 2011;184(4):392-394.

Carson: Are we sometimes harming patients by putting them through the rigors of weaning, when what they should be doing is recovering?

White: Probably. And I think that some of the pressure to wean patients off mechanical ventilation is driven by convention. You can get a patient to wean, sometimes, at tremendous cost to the patient, and it may be that that period of rest on mechanical ventilation is part of a recovery process.

MacIntyre: I'm not sure how that applies in this population. Weaning implies a gradual reduction, and many people think a gradual reduction to zero, but I think many of us would prefer to make it a reduction to a certain tolerable, comfortable level, and then if they're still not ready to go, just leave them there. As Dean and I and others have argued, periodicallyperhaps daily_ check them and see where they are. Are they ready to come off? That's the role of the SBT. But the idea that every day you have to keep trying to pull the level of ventilatory support down may cause more harm than good. In fact, what you ought to do is just keep them comfortable, reverse the reversible, and use the SBT not as a weaning tool but as an evaluation tool to see if they're ready.

Nelson: Is there a point in the weaning process when we can conclude that a patient with chronic critical illness is not going to wean from the ventilator, and come to terms with that conclusion? I do not think that question has a data-based answer yet. That would be extremely helpful information.

MacIntyre: We looked at the data at the NAMDRC [National Association for Medical Direction of Respiratory Care] consensus conference. There has been more reported since then, but the data at that time suggested that at the 90 day mark something like $95 \%$ or more of patients had come off the ventilator, and it seemed like that at that point maybe we'd gone as far as we could go with a patient, and they're not going to get any better. There are extenuating circumstances, of course.

White: There are, and you always have to be aware of them. But when you go through the literature and look at weaning times and take the 75 th percentile of the data given, it seems that somewhere in the region of 60 to 90 days is where failure to wean is being defined.

King: You mentioned speaking valves. I recently met with some physicians in France who are abandoning speaking valves and going to ventilator manipulation: mainly increased PEEP. Are you also working with that? You said you found benefit with swallowing using a speaking valve. 
White: When you say abandoning the speaking valve, is that an in-line speaking valve with mechanical ventilation or over a tracheostomy?

King: With mechanical ventilation.

White: Using an in-line valve in somebody with a lot of secretions is very difficult. A lot of our patients have COPD, and they don't do well with an in-line speaking valve. So in that population sometimes we do use leak-speech, which I think is what you're referring to, where we bring the cuff down and augment the delivered tidal volume and allow some air to pass around the vocal cords.

King: They're increasing PEEP.

Hess: That's commonly what we do. We usually do not use a speaking valve to facilitate speech in a mechanically ventilated patient. I think that it is potentially dangerous, particularly in a patient with neuromuscular disease. It is potentially harmful if you put a speaking valve in-line and the patient obstructs their upper airway and cannot remove the device. The approach that we very commonly use is to let down the cuff, increase the PEEP in order to increase the amount of leak to the upper airway, and use that to facilitate speech. We also make some manipulations to tidal volume and inspiratory time.

In fact, some of the people I work with, and others, have written several papers showing that the quality of speech can be just as good as with a speaking valve. ${ }^{1,2}$ I personally think it's much safer to avoid a speaking valve in mechanically ventilated patients. I'm a big fan of facilitating speech in the patient with a tracheostomy, ${ }^{3}$ but I am not a big fan of the speaking valve in mechanically ventilated patients.

1. Hoit JD, Banzett RB, Lohmeier HL, Hixon TJ, Brown R. Clinical ventilator adjustments that improve speech. Chest 2003;124(4): 1512-1521.

2. Prigent H, Garguilo M, Pascal S, Pouplin S, Bouteille J, Lejaille M, et al. Speech effects of a speaking valve versus external PEEP in tracheostomized ventilator-dependent neuromuscular patients. Intensive Care Med 2010; 36(10):1681-1687.

3. Hess DR. Facilitating speech in the patient with a tracheostomy. Respir Care 2005;50(4): 519-525.

White: We rarely use the in-line speaking valves, and when we do, it's usually on the patient's demand. It's a very small number of people that we use them on, and we've moved away from it over the years.

Muldoon:* When thinking through a weaning strategy for a PMV patient, how important is it to differentiate whether their primary failure is hypoxemic or hypercapnic?

White: Well, the hypoxemic patient is more likely to be somebody with parenchymal disease, possibly. Those patients are more likely to resolve that

* Sean R Muldoon MD MPH, Kindred Healthcare, Hospital Division, Louisville, Kentucky. over a period of time and maybe move back to a weaning strategy. In other words, patients who have a widened alveolar-arterial oxygen difference are different from those with an elevated $\mathrm{CO}_{2}$ respiratory failure. Obviously, the COPD population can have components of both. Are you asking whether that is something we should use to change our weaning strategy?

Muldoon: My observation is that we lump them together, and so the question is whether there is a difference between primarily pump failure/diaphragmatic weakness and a muscle training strategy, and the hypoxemic group where there's a different approach? Or should we lump them together?

White: I think that how you remove the patient from the mechanical ventilator is going to be based on the strategies I outlined: removing the barriers and having a systematic way of evaluating the patient on a regular basis. The subtleties of the individual causes of respiratory failure, as I alluded to, are important to know, because the timing of that weaning is going to be different. If you have a slow-to-resolve ARDS, which may be more of a hypoxemic problem, it'd be very different from somebody with COPD. Peter Bagley, in a paper published many years ago, showed that. ${ }^{1}$

1. Bagley PH, Cooney E. Community-based regional ventilator weaning unit: development and outcomes. Chest 1997;111(4): 1024-1029.

This article is approved for Continuing Respiratory Care Education credit. For information and to obtain your CRCE

(free to AARC members) visit

www.RCJournal.com 